\title{
A diagnosis more than skin deep: A case of late-stage pulmonary langerhans' cell histiocytosis with skin lesions
}

\author{
Joshua Verson, Alexander Egusquiza, David C Nguyen \\ School of Medicine, Rush University Medical Center, Chicago, Illinois, USA
}

Received: November 13, 2016

Accepted: January 9, 2017

Online Published: January 20, 2017

DOI: $10.5430 /$ crim.v4n1p68

URL: https://doi.org/10.5430/crim.v4n1p68

\begin{abstract}
Introduction: Langerhans' cell histiocytosis $(\mathrm{LCH})$ is a group of rare diseases with a wide variety of presentations across age groups. We describe a case of pulmonary LCH diagnosed at a late stage despite having additional skin findings for several years. Case description: A 32-year-old male presented with acute exacerbation of chronic dyspnea resulting in markedly decreased functional capacity as well as cutaneous lesions. He had smoked cigarettes for 10 years with no occupational exposures. The patient also complained of a pruritic rash in his axillary and groin areas. His vitals were within normal limits. His exam was notable for diminished right lung sounds and expiratory wheezes bilaterally. A chest x-ray showed marked emphysema and bilateral upper lobe reticular opacities suggestive of scarring. Computerized tomography of the chest showed loculated right pneumothorax and hyperinflated lungs with destructive changes secondary to numerous irregular, thick walled cysts and scattered irregular pulmonary nodules. Diagnosis was ultimately confirmed with biopsy of concomitant cutaneous lesions and characteristic radiographic findings on Chest CT. The patient started inhaled corticosteroids, inhaled anticholinergic, and inhaled short-acting beta agonists. He was sent home with oxygen and strongly encouraged to abstain from smoking.

Discussion: LCH is characterized by end-organ infiltration of proliferating monoclonal Langerhans' cells, a histiocyte involved in antigen presentation. The highest incidence of $\mathrm{LCH}$ is between ages 5-10 years; the adult incidence is estimated to be less than half as frequent than the pediatric, about one patient in 500,000. LCH most commonly involves the skin, bones, and lungs. Pulmonary LCH (PLCH) is interesting in that it has a peak incidence in ages 20-40 years and occurs almost exclusively in smokers (>90\% of cases).

Conclusion: Smoking cessation is the cornerstone of management of PLCH. It has been hypothesized that tobacco smoke can incite the production of cytokines by alveolar macrophages, leading to increased recruitment of histiocytes to the lung parenchyma. In isolated pulmonary pathology, lung transplant with smoking cessation is often curative.
\end{abstract}

Key Words: Langerhans' cell histiocytosis, Histiocyte, Emphysema, Pneumothorax, Cysts, Pulmonary nodules, Skin lesions

\section{INTRODUCTION}

Langerhans' cell Histiocytosis ( $\mathrm{LCH}$ ) refers to a collection of rare diseases involving the infiltration of various organs by histiocytes (dendritic cells and macrophages) which are a part of the mononuclear phagocytic family. ${ }^{[1]}$ LHC can be classified as unifocal or multifocal and is most commonly encountered in the pediatric population. Adult presentations are far less common (est. 1:560,000 of the general popula-

*Correspondence: Joshua Verson; Email: Joshua_Verson@rush.edu; Address: Rush University Medical Center, Chicago, Illinois, USA. 
tion) and are most often found in the bone, skin, and lungs. ${ }^{[1]}$ Lung involvement is very common in the disease, referred to as Pulmonary Langerhans' Cell Histiocytosis wide variety of presentations across age groups. Consequently, the diagnosis is often difficult to make.

We describe a case of pulmonary $\mathrm{LCH}$, which was eventually diagnosed at a late stage despite having additional skin findings for several years. This case highlights the importance of consideration of diagnoses that can explain seemingly separate findings.

\section{CASE DESCRIPTION}

A 32-year-old male presented with acute exacerbation of chronic shortness of breath. He had reported that he could not walk more than 1 block before needing to rest. He had no notable occupational exposures. He had smoked cigarettes for 10 years.
Four years prior, the patient had a spontaneous pneumothorax. He had a bilateral video-assisted thoracic surgery done for removal of lung blebs found at that time. No lung tissue studies had been done. Around the same time, the patient started developing plaque-like skin lesions in his axilla and inguinal folds. There was occasional scant green drainage from these lesions. They had been treated with antibiotics in the past but never fully remitted.

Patient was saturating $92 \%$ on 2 liters of supplemental oxygen via nasal cannula. The rest of his vitals were within normal limits. His exam was notable for diminished breath sounds in the right mid and apical lung fields as well as expiratory wheezes and coarse crackles bilaterally. There were well-demarcated violaceous to erythematous plaques with hyperpigmented borders in the bilateral axilla and groin region. The right axillary plaque appeared mildly infiltrative, while the remaining plaques revealed central maceration and areas of focal erosion (see Figure 1).
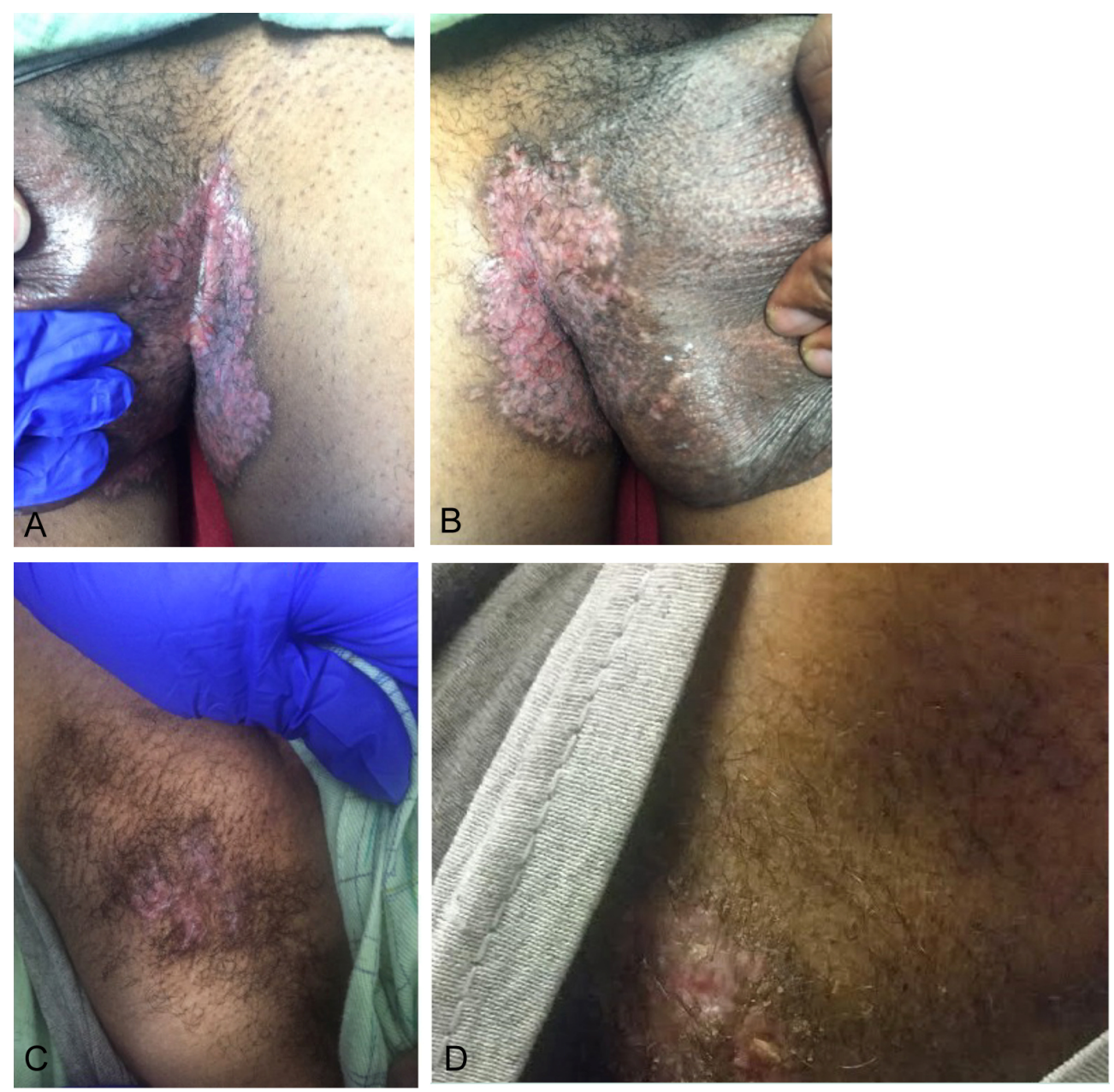

Figure 1. Dermatological findings. A. Left inguinal fold; B. Right inguinal fold; C. Right axilla; D. Left axilla 
Further work up consisted of cutaneous punch biopsies of the skin lesions. Pathology report demonstrated an infiltrate of histiocytic cells admixed with lymphocytes within the papillary dermis. Clusters of histiocytic cells were also present within the overlying epidermis. Immunohistochemical stains for S100 protein and CD1a were positive within these cells supporting the diagnosis of Langerhans Cell Histiocytosis. The patient was started on steroid burst with prednisone and switched to inhaled corticosteroid. Ambulatory saturations performed on and off supplemental oxygen with patient desaturating $<88 \%$ on RA. At time of discharge, patient was transitioned to Qvar, Spiriva twice daily and albuterol inhaler as needed. He was discharged with home oxygen with plan for follow up in pulmonary clinic for outpatient Pulmonary Function Tests. Smoking cessation was discussed at length with the need for possible lung transplantation in the future.

A chest $\mathrm{X}$-ray performed in emergency department showed marked emphysema and bilateral upper lobe reticular opacities suggestive of scarring. This was followed up with computerized tomography of the chest showing hyperinflation of the lungs with advanced destructive changes of the bilateral parenchyma, most pronounced at the bilateral apices. Innumerable irregular, slightly thick walled cysts are seen throughout the bilateral lungs, with a few irregular pulmonary nodules noted, particularly in the right lower lobe, most compatible with Langerhans cell histiocytosis (see Figure 2).

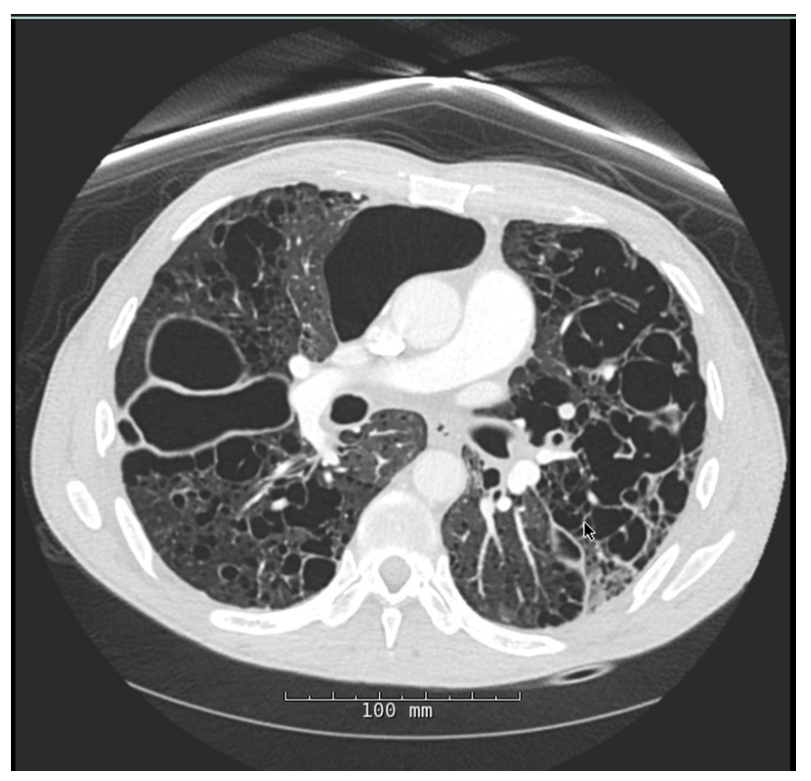

Figure 2. CT Scan

\section{Discussion}

$\mathrm{LCH}$ is characterized by end-organ infiltration of proliferating monoclonal Langerhans' cells, a histiocyte involved in antigen presentation often affecting lungs, bones. ${ }^{[1]}$ The highest incidence of LCH is between ages 5-10 years; the adult incidence is estimated to be less than half as frequent than the pediatric. LCH can have a diverse array of presentations but most commonly involve the skin, bones, and lungs. Pulmonary LCH (PLCH) is interesting in that it has a peak incidence in ages 20-40 years and occurs almost exclusively in smokers (>90\% of cases). ${ }^{[2]}$ Spontaneous pneumothorax has been reported as the presenting finding in 10\%-20\% of cases. Co-occurring extra-pulmonary findings have been reported in 5\%-10\% of cases of PLCH with skin findings being the most common.

Epidemiologically, the prevalence and incidence of PLCH is unknown, but studies of a variety of lung-biopsy specimen of patients with different forms of interstitial lung disease identified PLCH in about 5\%. ${ }^{[3]}$ There are no known genetic factors predisposing people to $\mathrm{PLCH}$ and for reasons not completely understand PLCH occurs more often in Caucasians. The only consistent epidemiological association is with cigarette smoking, with over $90 \%$ of patients having some form of smoking in their past. ${ }^{[4]}$

Histologically, the Langerhan's cell is distinguished based on a pale staining cytoplasm and Birbeck granules on on electron microscope. On pathogenesis, they exhibit positive immunohistochemical staining S100 protein. Langerhans cell also exhibits the presence of CD1a antigen staining on the surface of cells, which are not present on any other histiocytic cells. The most recent hypothesis regarding the association between smoking and PLCH on a cellular level occurs as bombesin peptides are secreted from neuroendocrine cells which are increased in smokers, and may play a role in mediating lung injury through the promotion of cytokines (IL-2). ${ }^{[5]}$ Tobacco smoke can additionally incite the production of cytokines by alveolar macrophages and fibroblasts, leading to increased recruitment of histiocytes to the lung parenchyma.

As was done with our patient, smoking cessation is the cornerstone of management of PLCH. Smoking cessation leads to stabilization of symptoms, and in some reports, objective radiologic and physiologic improvement in lung function. ${ }^{\text {[5] }}$ Immunosuppressive therapies such as glucocorticoids and cytotoxic medications which were given to our patient have been shown in studies to have a very limited benefit in the improvement of dermatological manifestations but are often used empirically to treat symptomatic patients with nodular lesions with the end goal of resolving granulomatous and inflammatory processes. ${ }^{[5]}$ One study demonstrated corticosteroid use of $0.5-1 \mathrm{mg} / \mathrm{kg} /$ day of prednisone to be associated with clinical and radiological improvement but no change in overall lung function, ${ }^{[2]}$ while a separate retrospective study 
demonstrated a deterioration in the patients treated with corticosteroids compared to untreated patients. ${ }^{[6]}$ In isolated pulmonary pathology in patients with severe respiratory deficiency, lung transplant with smoking cessation is often the only curative method available.

Prognostic factors include a 5-year survival estimate of greater than $75 \%^{[5]}$ with a median survival time of 12 years. ${ }^{[5]}$ The progression of PLCH is variable between patients due the rarity of its occurrence and the complicated progress of its course. Prognostic factors can range from spontaneous remission to end-stage fibrotic disease depending on whether or not the subjects abstain from cigarette smoking. ${ }^{[7]}$ A small study conducted by Mogulcok et al. ${ }^{[7]}$ followed two patients with proven $\mathrm{PLCH}$ in whom radiographic improvement of lung nodules after smoking cessation. Both patients had 23 and 25 pack-year smoking histories and within 3 months of smoking cessation, FEV1/FVC ratios improved from $82 \%$ to $92 \%$ in the first patient, and nearly complete resolution of extensive nodularity observed on initial HRCT scan. ${ }^{[7]}$ The second patient with histologically diagnosed PLCH demonstrated on chest radiography to have multiple nodular shadows of $3-10 \mathrm{~mm}$ in the upper lobes confirmed by HRCT and a FEV1/FVC of $98 \%$. After 6 months of smoking cessation there was complete radiographic resolution of nodules and pulmonary symptoms had disappeared. ${ }^{[7]}$ Of course the pitfall of this study is the small patient population, but due to the rarity of the disease and the resolution of clinical and radiographic symptoms, even small studies are extremely significant. If smoking cessation can truly lead to the resolution of PLCH in even two patients with histologically proven disease, it is imperative the physicians do everything within their power to convince patients to quit smoking tobacco products.

\section{Conclusion}

Langerhan's Cell Histiocytosis is a diagnosis that is inherently difficult to make due to its rarity and diversity of presentations. However, our patient fits the classic demographic for PLCH and manifestations that were not uncommon in previously described cases. An investigation into potential unifying explanations of seemingly separate findings in the year's prior could have lead to earlier diagnosis. Smoking cessation was advised but it is unclear the extent of benefit to the patient at this stage. Corticosteroids and chemotherapeutic medications have also shown to benefit the patient but it is unclear to what extent and at what point in the progression of the disease in which the medications demonstrate positive effects. Larger studies need to be performed to better understand the beneficence of treatment options.

\section{CONFLicts OF INTEREST Disclosure}

The authors have no competing interests to declare.

\section{REFERENCES}

[1] Ciuche A, Nistor C, Pantile D, et al. Spontaneous pneumothorax in a case of pulmonary Langerhans Cell Histiocytosis. Mædica. 2011; 6(3): 204-209.

[2] Schönfeld N, Frank W, Wenig S, et al. Clinical and radiologic features, lung function and therapeutic results in pulmonary histiocytosis X. Respiration. 1993; 60(1): 38. PMid:8469818 https : //doi.org/10.1159/000196171

[3] Aguayo SM, King TE Jr, Waldron JA Jr, et al. Increased pulmonary neuroendocrine cells with bombesin-like immunoreactivity in adult patients with eosinophilic granuloma. J Clin Invest. 1990; 86: 838. PMid:2394833 https://doi.org/10.1172/JCI114782

[4] Vassallo R, Ryu JH, Colby TV, et al. Pulmonary Langerhans' cell histiocytosis. NEJM. 2000; 342(26): 1969-1977. PMid:10877650 https://doi.org/10.1056/NEJM200006293422607

[5] Tazi A. Adult pulmonary Langerhans' cell histiocytosis. Eur Respir J. 2006; 27: 1056-1065. PMid:16772390 https ://doi .org/10.1 $183 / 09031936.06 .00024004$

[6] Delobbe A, Durieu J, Duhamel A, et al. Determinants of survival in pulmonary Langerhans' cell granulomatosis (histiocytosis X). Eur Respir Journal. 1996; 9: 2002-2006. PMid:8902457 https://doi.org/10.1183/09031936.96.09102002

[7] Mogulkoc N, Veral A, Bishop PW, et al. Pulmonary Langerhans' cell histiocytosis: radiologic resolution following smoking cessation. Chest. 1999; 115: 1452. PMid:10334170 https ://doi.org/10.1 $378 /$ chest.115.5.1452 